\title{
Cell type-specific gene expression patterns associated with posttraumatic stress disorder in World Trade Center responders
}

\author{
Pei-Fen Kuan ${ }^{1}$, Xiaohua Yang ${ }^{2}$, Sean Clouston $\mathbb{0}^{3}$, Xu Ren ${ }^{1}$, Roman Kotov ${ }^{4}$, Monika Waszczuk ${ }^{4}$, Prashant K. Singh ${ }^{5}$, \\ Sean T. Glenn ${ }^{5}$, Eduardo Cortes Gomez $\mathbb{1}^{6}$, Jianmin Wang ${ }^{6}$, Evelyn Bromet ${ }^{4}$ and Benjamin J. Luft ${ }^{2}$
}

\begin{abstract}
Posttraumatic stress disorder (PTSD), a chronic disorder resulting from severe trauma, has been linked to immunologic dysregulation. Gene expression profiling has emerged as a promising tool for understanding the pathophysiology of PTSD. However, to date, all but one gene expression study was based on whole blood or unsorted peripheral blood mononuclear cell (PBMC), a complex tissue consisting of several populations of cells. The objective of this study was to utilize RNA sequencing to simultaneously profile the gene expression of four immune cell subpopulations (CD4T, CD8T, B cells, and monocytes) in 39 World Trade Center responders (20 with and 19 without PTSD) to determine which immune subsets play a role in the transcriptomic changes found in whole blood. Transcriptome-wide analyses identified cell-specific and shared differentially expressed genes across the four cell types. FKBP5 and PI4KAP1 genes were consistently upregulated across all cell types. Notably, REST and SEPT4, genes linked to neurodegeneration, were among the top differentially expressed genes in monocytes. Pathway analyses identified differentially expressed gene sets involved in mast cell activation and regulation in CD4T, interferon-beta production in CD8T, and neutrophilrelated gene sets in monocytes. These findings suggest that gene expression indicative of immune dysregulation is common across several immune cell populations in PTSD. Furthermore, given notable differences between cell subpopulations in gene expression associated with PTSD, the results also indicate that it may be valuable to analyze different cell populations separately. Monocytes may constitute a key cell type to target in research on gene expression profile of PTSD.
\end{abstract}

\section{Introduction}

Posttraumatic stress disorder (PTSD) is a complex disorder that affects $\sim 7 \%$ of the US population ${ }^{1}$. PTSD develops in response to exposure to traumatic events and is characterized by emotional numbing, intrusive memories, avoidance, and hyperarousal ${ }^{2}$. PTSD can lead to cognitive, social and occupational impairment, and is associated with neurodegeneration ${ }^{3,4}$ thus causing substantial social and economic burden for individuals

\footnotetext{
Correspondence: Benjamin J. Luft (Benjamin.luft@stonybrookmedicine.edu) 'Department of Applied Mathematics and Statistics, Stony Brook University, Stony Brook, NY, USA

${ }^{2}$ Department of Medicine, Stony Brook University, Stony Brook, NY, USA Full list of author information is available at the end of the article.
}

affected by this condition ${ }^{5}$. The etiology of PTSD is not well understood. However, genetic studies involving twin, candidate gene, and genome-wide association analyses ${ }^{6}$ have revealed that heritability of PTSD is moderate in size (ranging from 30 to $40 \%)^{7,8}$, and that genetic factors may play an important role in the vulnerability to, and resilience following, trauma exposure in PTSD ${ }^{9}$. PTSD is also consistently associated with altered functioning of the immune system, including increased levels of circulating C-reactive protein and pro- and anti-inflammatory cytokines $^{10-12}$. To date, the link between genetic and immunologic processes in PTSD remains unclear.

Gene expression analyses can identify critical downstream biological process associated with genetic and 
epigenetic variations and thus can potentially inform efforts to identify biomarkers for PTSD ${ }^{13}$. Transcriptomewide gene expression profiling has emerged as the preferred approach for explicating gene regulation, because it allows for an unbiased investigation of expression patterns without a priori knowledge of genetic risk factors ${ }^{14}$. Taking such hypothesis-free approach, previous studies, including work from our group, identified differentially expressed pathways related to immune functions and inflammation to be most prominent in PTSD ${ }^{15-17}$.

As direct sampling of the brain is not feasible, most prior studies were performed using whole blood or unsorted peripheral blood mononuclear cell $(\mathrm{PBMC})^{13,15-22}$. To a large extent, gene expression patterns in blood are consistent with patterns observed in brain ${ }^{23,24}$, suggesting that the molecular signature of PTSD may be obtained outside the brain. An additional strength of focusing on blood tissue is its feasibility as a potential clinical biomarker. However, blood is a complex tissue that consists of several populations of cells, and each has a distinct gene expression profile. Alterations in immune regulatory networks are expected to have functional consequences primarily in certain subsets of immune cells. Thus, analyses of whole blood are likely to weaken the signal. Indeed, in autoimmune diseases, analyses of gene expression in specific cell types reveal stronger links to target disease than analyses of whole blood ${ }^{25-27}$. As PTSD is implicated in immune responses and suggestive evidence links it to autoimmune diseases ${ }^{28-31}$, studying genetic alterations in isolated immune cell subsets may provide a clearer understanding of the link between PTSD and immune function. Noting this, one previous case-control study examined gene expression in isolated monocytes in 49 men with and without PTSD ${ }^{32}$, and identified three significantly downregulated genes (PF4, SDPR, and HIST1H2AC) despite finding no evidence of chronic inflammation. The authors also found a large range of genes with clinically meaningful effect sizes that were predominantly under-expressed in PTSD, suggesting decreased gene activation in immune cells.

To our knowledge, no study of PTSD to date has profiled gene expression in isolated immune subsets other than monocytes. The current study addressed this gap by conducting a transcriptome-wide gene expression study using the state-of-the-art RNA sequencing (RNA-Seq) approach on RNA derived from the main subsets of isolated immune cells retrieved from peripheral blood. To this end, we analyzed blood from 39 male responders to the WTC disaster with and without WTC-connected PTSD whose whole-blood transcriptome had previously been studied ${ }^{15}$. We compared gene expression profiles within four isolated subsets of cells: $\mathrm{CD} 3+\mathrm{CD} 4+\mathrm{T}$ cells (CD4T), CD3+CD8+ T cells $(\mathrm{CD} 8 \mathrm{~T}), \mathrm{CD} 19+\mathrm{B}$ cells $(\mathrm{B}$ cells) and CD14+ monocytes (monocytes) to determine the immune subsets that were responsible for the changes in the transcriptome found in our previous study ${ }^{15}$. Also, we performed candidate gene analyses to test replicability of the only previous study of PTSD gene expression in monocytes, which identified downregulation of PF4, $S D P R$, and HIST1H2AC in this cell type. To better understand genetic predisposition to PTSD, biological pathway analyses were performed on differentially expressed genes. The results from isolated immune cells were compared to results obtained from PBMC and whole blood. This study tested the hypothesis that both common and distinct gene expression patterns in PTSD would be observed in immune cells relative to PBMC and whole blood.

\section{Methods \\ Setting}

The World Trade Center (WTC) disaster was a catastrophic event that simultaneously exposed tens of thousands of individuals, including WTC responders who worked on the site during rescue and recovery operations, to acute psychological and physical trauma ${ }^{33,34}$. Stony Brook University manages the second largest program and monitors responders residing on Long Island, $\mathrm{NY}^{35}$. Most responders in the Stony Brook program are male, worked as police, and were aged 39 in September 2001. We previously found that $18 \%$ of the cohort developed WTC-connected PTSD, and 10\% had chronic WTCPTSD $^{34}$.

\section{Participants and clinical assessment}

This study utilized blood samples from a subset of participants $(n=39)$ based on their polygenic expression scores from a previous study in which we had already demonstrated significant gene dysregulation ${ }^{15}$. Details on recruitment, clinical assessment, and biomarker sampling were described previously ${ }^{15}$. The 39 participants in the present study were non-smoking males (mean age $=51.4$, $\mathrm{SD}=8.94) ; 20$ had a history of DSM-IV WTC-PTSD determined using the Structured Clinical Interview for DSM-IV ${ }^{36}$ and had highest polygenic expression scores, while 19 did not and had lowest polygenic expression scores. Interviews were conducted concurrently to blood draw by professional interviewers under supervision of clinical psychologists.

\section{Peripheral blood mononuclear cell (PBMC) isolation and freezing}

Whole-blood samples were collected in BD Vacutainer $\mathrm{CPT}$ cell preparation tubes containing sodium heparin as the anti-coagulant (BD, Franklin Lakes, NJ, USA). The $\mathrm{CPT}$ tubes were processed within $2 \mathrm{~h}$ on at room temperature according to the manufacturer's instruction. The 
purified PBMC cells were aliquoted in 90\% FBS $+10 \%$ dimethylsulphoxide (DMSO) with cell concentration at $>2 \times 10^{7}$ cells $/ \mathrm{ml}$ per cryovial $(0.75 \mathrm{ml}$ per cryovial $)$. To ensure stepwise temperature decrease ${ }^{37}$, the cryovial were placed in Mr Frosty' containers (Nalgene/Thermo Fisher, Rochester, NY, USA) with isopropyl alcohol medium in $-80^{\circ} \mathrm{C}$ freezers for $24-48 \mathrm{~h}$. The cryovials were transferred quickly into liquid nitrogen for long term storage. The crypreserved PBMC samples were shipped with a dry-ice package to the Roswell Park Cancer Center and stored at $-8^{\circ} \mathrm{C}$ for flow sort sub-cell separation and RNA extraction within $1-2$ weeks.

\section{Flow sorts on frozen PBMC, total RNA isolation, library preparation, and sequencing}

Frozen PBMC were thawed, restored, washed, and counted. An aliquot of cells was resuspended in PBS containing 0.1\% BSA (PBS/BSA), blocked with mouse IgG, and labeled at room temperature with the mouse anti-human antibodies. The cells were then washed in PBS/BSA, resuspended in $1 \mathrm{ml} \mathrm{PBS/BSA}$, filtered through a 35 micron mesh, and diluted with $1 \mathrm{ml}$ sort buffer. Four-way sorting was performed on a BD FACSAria II sorter, where monocytes, B cells, CD4T, and CD8T cells were sorted simultaneously. The purification of total and small RNA was prepared using the miRNeasy mini kit (Qiagen). Quantitative assessment of the purified total RNA was accomplished using a Qubit Broad Range RNA kit (Thermo Fisher), which was then qualitatively evaluated by a 2100 Bioanalyzer (Agilent technologies). The sequencing libraries were prepared with the TruSeq Stranded Total RNA kit (Illumina Inc) and sequenced on a HiSeq2500 sequencer using a 100 cycle single-read cluster kit. Additional details are provided in Supplementary Materials.

\section{RNA-Seq data preprocessing}

Alignment was performed using the TopHat2 software s $^{38}$ which utilizes Bowtie2 ${ }^{39}$ (http://bowtie-bio.sourceforge. net/bowtie2/index.shtml) on RefSeq (NCBI Reference Sequence Database) annotation and human reference genome (GrCh37-hg19 version) ${ }^{40}$. Spliced alignment of the reads to the reference genome was done with the TopHat2 software allowing a maximum of one mismatch per read; quality control was done using RSeQC. Other genomic related data were obtained using UCSC's genome repository. ${ }^{41}$ Quality control for raw reads was performed with FastQC, ${ }^{42}$ and adapter trimming was done with cutadapt $^{43}$. The number of read counts mapping to each gene was computed using htseq-count. ${ }^{44}$

\section{Estimation of batch effects}

The potential for batch effects was estimated from the log-normalized gene counts data using surrogate variable analysis approach for sequencing data (svaseq) ${ }^{45}$. The estimated surrogate variables were included in differential expression analyses as adjustment factors. Proportions of CD4T, CD8T, monocytes and B cells estimated from cell sorting were included in differential expression analysis for PBMC and whole blood as adjustment factors. We also compared the estimated cell subsets from two computational tools, namely CIBERSORT ${ }^{46}$ and $\mathrm{xCell}^{47}$ software. CIBERSORT was developed based on deconvolution method using microarray datasets, whereas xCell was based on integration of gene set enrichment analysis and deconvolution method on both RNA-Seq and microarray datasets. The estimated cell type abundances between PTSD and non-PTSD were compared using the two sample $t$-tests.

\section{Differential expression analysis}

Differential expression analyses of RNA-Seq data generated from isolated CD4T, CD8T, B cells, and monocytes was performed using DESeq ${ }^{48}$ software based on multivariable-negative binomial generalized linear models that adjusted for age, race, and estimated surrogate variables to account for potential batch effects. Genes with low expression were filtered using the cpm (count-permillion) function in edge $\mathrm{R}^{49}$. A total of 15,947 genes were included in the analysis after filtering. Statistical significance was assessed via the Wald test using appropriate contrasts to identify differentially expressed genes associated with PTSD for each cell type. A false discovery rate $(\mathrm{FDR})^{50}$ control was used to account for multiple testing. FDR $<0.05$ was used to identify statistically significant genes. Post hoc analysis comparing the number of differentially expressed genes at a range of nominal $p$-value thresholds was conducted to evaluate the signal strength of differential expression associated with PTSD across cell types. A joint analysis, adjusting for cell types and the confounders described above was also conducted to identify differentially expressed genes associated with PTSD. Heatmaps and volcano plots were used to visualize gene expression patterns. Principal component analysis (PCA) was performed on the matrix of normalized gene counts. The first three principal components (PC1, PC2, and PC3) were used to visualize global gene expression patterns. Pearson correlation coefficients were computed on the estimated $\log 2$ fold change from DESeq2 in order to conduct post hoc comparisons of similarities and differences across cell types.

\section{Candidate gene analysis}

The association between PTSD and gene expression was examined for previously implicated genes in monocytes $(P F 4, S D P R, H I S T 1 H 2 A C)^{32}$. The estimated $\log 2$ fold change, nominal and Bonferroni adjusted $p$-values for these three genes were reported. 
Table 1 Clinical characteristics of the 39 samples

\begin{tabular}{|c|c|c|c|}
\hline All & Case $N=20$ & Control $N=19$ & $P$-value \\
\hline \multicolumn{4}{|l|}{ Age } \\
\hline Mean (SD) & $53.35(8.12)$ & $49.37(9.51)$ & 0.169 \\
\hline \multicolumn{4}{|l|}{ Race N (\%) } \\
\hline Caucasian & $17(85)$ & 19 (100) & 0.231 \\
\hline Other & $3(15)$ & $0(0)$ & \\
\hline \multicolumn{4}{|l|}{$P C L$} \\
\hline Mean (SD) & $56.00(8.43)$ & $18.01(1.54)$ & $<0.01$ \\
\hline \multicolumn{4}{|c|}{ Polygenic score } \\
\hline Mean (SD) & $0.46(0.18)$ & $0.30(0.12)$ & $<0.01$ \\
\hline \multicolumn{4}{|l|}{$B M I$} \\
\hline Mean (SD) & $31.90(5.31)$ & $32.06(7.32)$ & 0.937 \\
\hline \multicolumn{4}{|l|}{ LRS N (\%) } \\
\hline Yes & $14(70)$ & $8(42)$ & 0.111 \\
\hline No & $6(30)$ & $11(58)$ & \\
\hline \multicolumn{4}{|l|}{ Exposure } \\
\hline Mean (SD) & $2.06(0.97)$ & $1.60(0.98)$ & 0.195 \\
\hline \multicolumn{4}{|l|}{ GERD N (\%) } \\
\hline Yes & $17(85)$ & $11(58)$ & 0.082 \\
\hline No & $3(15)$ & $8(42)$ & \\
\hline
\end{tabular}

The $p$-values were computed from $t$-test (for continuous variables) and Fisher's exact test (for categorical variables). The polygenic scores were computed based on our previous paper on whole blood

\section{Pathway and gene ontology analyses}

Pathway and gene ontology analyses were carried out using the over-representation via the Bioconductor package clusterProfiler ${ }^{51}$. Over-representation analysis was performed on the top 100 genes ranked by $p$-values from the differential expression analysis using the function enrichGO. In total, 5414 gene ontologies, including biological processes, molecular functions, and cellular components (the range of genes per gene set was 15-500) were tested. Statistically significant gene sets were corresponded to those with FDR $<0.05$ from overrepresentation analyses.

Discriminant analysis via elastic net regularized regression

To evaluate the synergistic effect of multiple genes in discriminating PTSD status within each cell type, we randomly divided the 39 samples into 29 training and 10 test samples. The objective of this analysis is to provide another analytic framework to compare the signal-tonoise ratio of the different cell types. Within the training set, the elastic net ${ }^{52}$ algorithm with threefold crossvalidation was applied to the top 1000 genes identified from the differential expression analyses of the current training set to ensure unbiased selection of candidate features. The area under receiver operating curve (AUC), based on the model obtained from the training set, was computed on the test set. The random partitioning of training and test samples was repeated ten times, and the average AUC of the test set was reported.

Additional statistical analyses including weighted gene co-expression network analysis ${ }^{53}$ and differential expression analysis comparing each cell type pair stratified by PTSD status are provided in Supplementary Materials. An overview of the cell-specific RNA-Seq data analysis pipeline was given in Supplementary Figure 1.

\section{Code availability}

Script for the main analysis is available at http://www. ams.sunysb.edu/ pfkuan/CellGeneExp.

\section{Results}

Participant characteristics

We did not find significant group difference on the exposure severity between cases and controls. Furthermore, we compared controls and PTSD cases on hallmark WTC-related disorders: lower respiratory symptoms (LRS) and gastroesophageal reflux disease (GERD) symptoms, and did not find a group difference. Finally, the two groups did not differ on BMI, and demographic variables (Table 1).

\section{Differentially expressed genes across different cell types}

Principal component analysis (PCA) of transcriptomewide normalized gene expression counts (Fig. 1a-c) showed significant cell type differences, with CD4T and CD8T showing the largest degree of similarity. The PCA plot also showed that most of the gene expression changes observed were related to the specific cell types instead of PTSD status. Similar patterns were also observed when we restricted the comparison to the top 1000 most variable genes across the different cell types (Supplementary Figure 4A) or within each cell type (Supplementary Figure 4B-E). As expected, gene expression of unsorted PBMC fall in between that of the cell types. Transcriptome-wide estimated $\log 2$ fold change from DESeq2 for the differential expression analysis comparing WTC-PTSD to unaffected responders also showed that CD4T and CD8T were most similar to each other $(r=0.37, p<0.001)$ followed by B cells, whereas monocytes and whole blood were least similar $(r=0.061, p<0.001)$ (Fig. 2a). Correlations between differential gene expression profiles in whole blood and in immune cell types ranged between 0.06 and 0.11 . The top 100 genes identified within CD4T showed correlations ranging from 0.35 to 0.72 with other cell types. The top 100 genes identified from unsorted PMBC correlations ranging from 0.49 to 0.74 with the isolated immune subsets and 0.33 with whole blood. The 

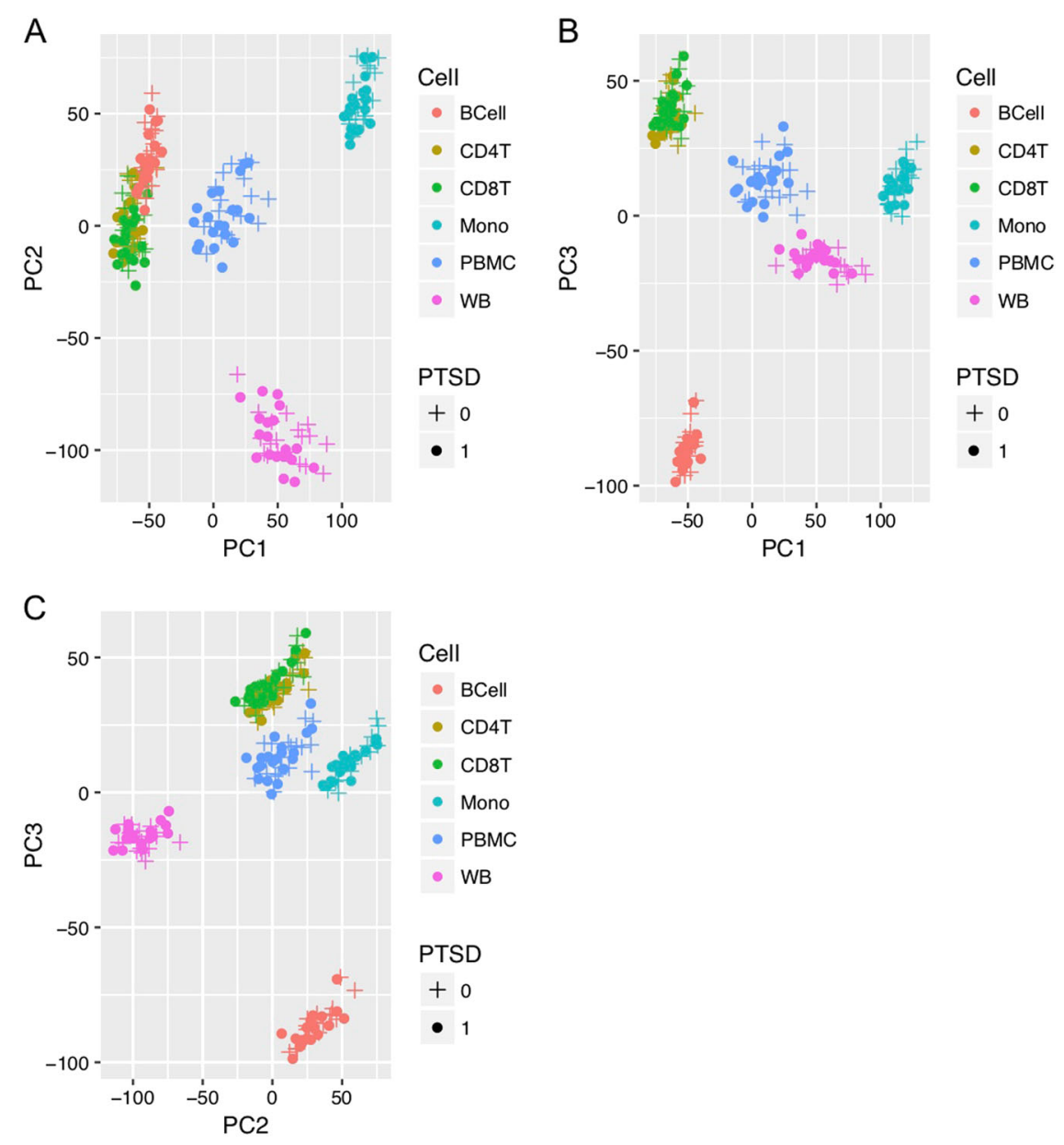

Fig. 1 Principal component analysis (PCA) of transcriptome-wide normalized gene expression counts assessing the difference in immune cell types. Each dot represented a sample, color coded by cell type (Red: B cells, Sage: CD4T, Green: CD8T, Turquoise: monocytes, Sky blue: PBMC, purple: whole blood, plotting symbol+: control, solid circle: PTSD). a $x$-axis denotes the value of PC1, $y$-axis denotes the value of PC2. $\mathbf{b} x$-axis denoted the value of PC1, $y$-axis denotes the value of PC3. c x-axis denoted the value of PC2, $y$-axis denotes the value of PC3

top 100 genes identified from whole blood showed correlations ranging from 0.036 to 0.2 with other cell types (Fig. 2b). These results suggested that the transcriptomewide differential gene expression observed in whole blood was partially attributed to differential gene expression in granulocytes that were removed during the separation of PBMC from whole blood.

The proportion of CD4T estimated from cell sorting was different between case and control ( $p$-value 0.019, Supplementary Figure 3A). Similar pattern of elevated CD4T abundances in PTSD was also observed using the cell subtypes estimates from CIBERSORT and xCell, although they did not meet statistical significance (Supplementary Figure 3B, 3C). The correlations between the cell subtype estimates from CIBERSORT and cell sorting were $>0.75$ for all cell types except CD8T (Supplementary Figure 3D); whereas, the correlations between $\mathrm{xCell}$ and cell sorting were lower in these cell types, except for $B$ cells. In addition, the estimated log2 fold change and the negative $\log p$-values from the differential expression analysis comparing PTSD to control, adjusting for cell heterogeneity using cell sorting proportions exhibit a higher correlation with CIBERSORT for both the unsorted PBMC and whole-blood analysis, compared to the adjustment using xCell (Supplementary Figure 8). CIBERSORT outputs the estimated proportions, which were directly comparable to the output of cell sorting. On the other hand, $x$ Cell outputs enrichment scores for the cell types, which may not be interpreted as proportions.

Volcano plots (Supplementary Figure 5A-D) depicting global differential gene expression patterns indicated an approximately equal amount of over- and underexpression comparing WTC-PTSD to non-PTSD across the four cell types. At FDR $<0.05,3,5,6,12$, and 3 genes were identified to be differentially expressed in CD4T, CD8T, monocytes, B cells, and unsorted PBMC, 

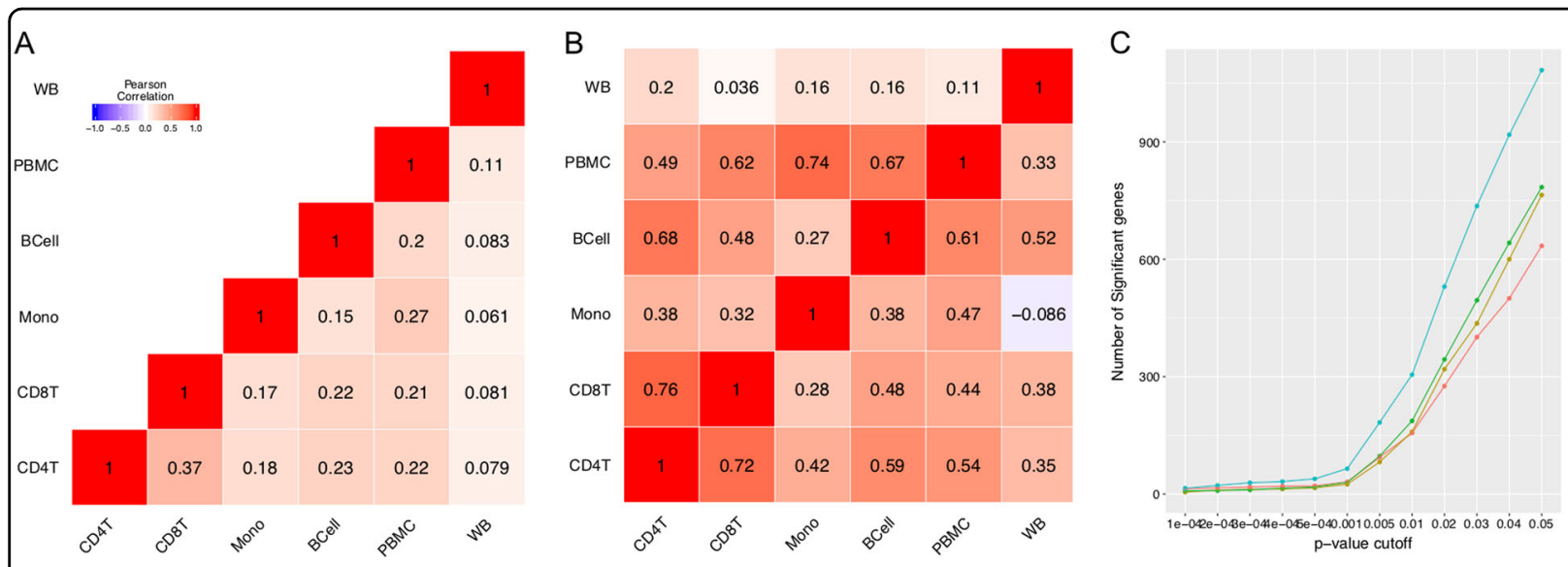

Fig. 2 Differential expression analysis. a Pairwise correlation coefficients between cell types comparing the estimated log2 fold change across all genes from DESeq2 for the differential expression analysis in PTSD. b Pairwise correlation coefficients of the estimated log2 fold change for the top 100 genes from DESeq2 for the differential expression analysis in PTSD for each cell type (row). The matrix is asymmetric because each row corresponds to different gene list, e.g., rows 1 is based on the top 100 differentially expressed (DE) genes in whole blood, whereas row 2 is based on top 100 DE genes in unsorted PBMC. c Number of differentially expressed genes in PTSD ( $y$-axis) at different nominal $p$-value thresholds ( $x$-axis) for each cell type

respectively (Fig. 3). Across different nominal $p$-value thresholds, monocytes identified the largest number of differentially expressed genes among the immune cell subsets (Fig. 2c), suggesting that monocytes contained the strongest gene expression differences in PTSD compared to CD4T, CD8T, and B cells.

Most of the genes identified to be statistically significant at FDR $<0.05$ were specific to each cell type (Fig. 3), except for PI4KAP1 which was upregulated in CD4T $($ FDR $<0.05)$ and the rest of the cell types with nominal $p$ values $<0.05$. In our previous study ${ }^{15}$, FKBP5 was found to be upregulated in whole blood in a sample of 282 responders. This gene remained consistently upregulated in this subset of 39 responders across all cell types (nominal $p$-values $<0.05$ in CD4T, CD8T, B cells, unsorted PBMC and whole blood, and nominal $p$-value $<0.1$ in monocytes). On the other hand, NDUFA1 and CCDC85B were previously found to be downregulated in whole blood. NDUFA1 exhibited weak downregulation effect sizes in all cell types (nominal $p$-values $>0.05$ ), whereas $C C D C 85 B$ did not reach statistical significance in this subset of 39 responders. The proportion of downregulated genes in monocytes vary between 40 and $50 \%$ across different nominal $p$-value thresholds (Supplementary Figure 6).

The joint differential expression analysis across the four cell subsets for PTSD status identified 34 genes at FDR $<$ 0.05 (Supplementary Figure 7). Among these 34 genes, only PI4KAP1 was in common with the cell-specific gene expression analysis of CD4T. FKBP5 was among these 34 genes and was upregulated in the joint analysis, consistent with our previous findings.

\section{Candidate gene analysis}

As noted above, PF4, SDPR, HIST1H2AC were previously identified to be downregulated in monocytes in male subjects with PTSD using microarrays ${ }^{32}$. Among these three genes, PF4 $(p=0.01$, Bonferroni $p=0.029)$ was also found to be at least marginally downregulated in monocytes in this study. Results also extended to other cell types and whole blood (Fig. 3). PF4 was also significantly downregulated (FDR $<0.05)$ in the joint differential expression analysis of the four cell types for PTSD status (Supplementary Figure 7).

\section{Pathway and gene ontology analyses}

Pathway and gene ontology analyses identified 18, 11, and 9 gene sets to be enriched at FDR $<0.05$ among the top 100 genes associated with PTSD in CD4T, CD8T, and monocytes, respectively (Fig. 4). No gene set was significant at FDR $<0.05$ for $\mathrm{B}$ cells. Most of the enriched gene sets were related to immune responses and inflammation. Gene sets related to mast cell activation and regulation emerged as the top ontology for CD4T. Gene sets related to interferon-beta production were identified as the top ontology for CD8T, whereas neutrophil-related gene sets were the top ontology for monocytes.

\section{Cell-specific discriminant analysis for PTSD}

The average AUC values over the 10 random splits for CD4T and monocytes were 0.666 and 0.735 , respectively; whereas, the average AUC values for CD8T and B cells were $\sim 0.5$ (equivalent to random guess). Despite the small sample size, monocytes showed promising discriminant power in differentiating PTSD from healthy controls. This 


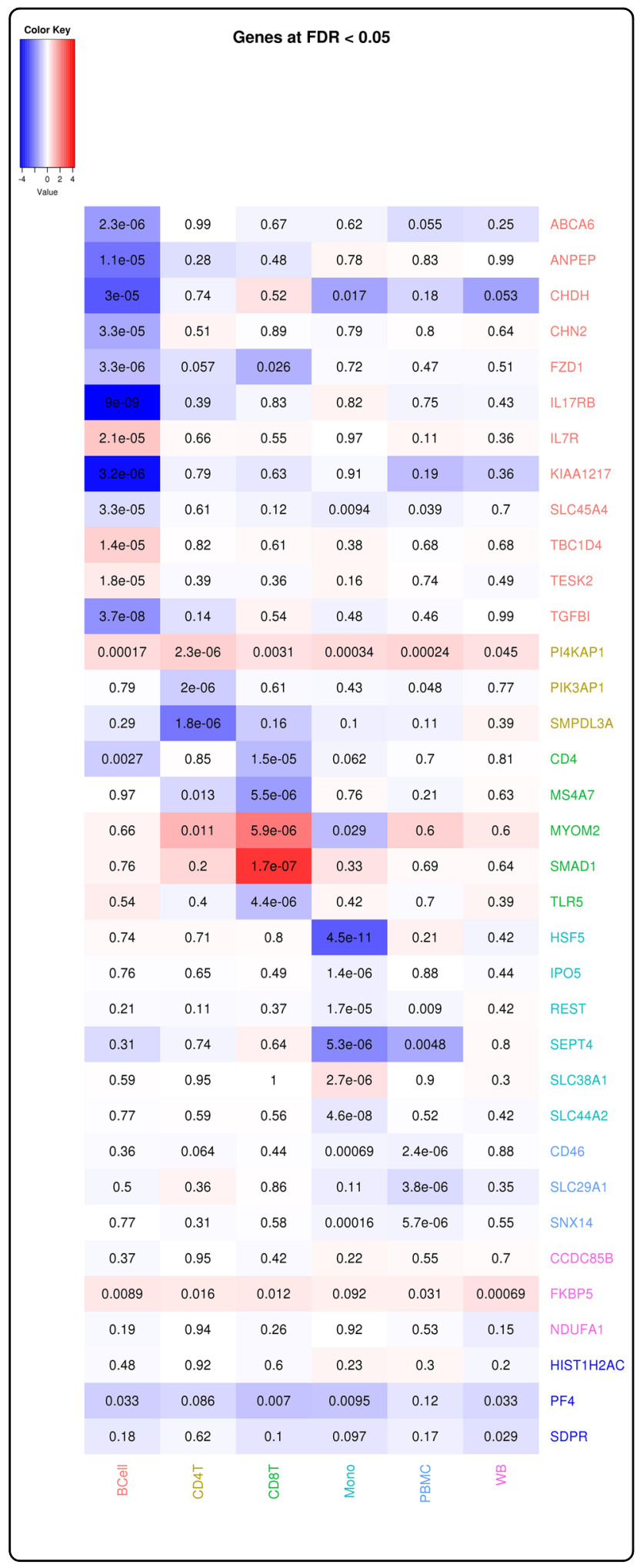

result was also consistent with the earlier finding that at a fixed $p$-value threshold, a higher proportion of differentially expressed gene in PTSD was detected in monocytes compared to other immune cell types.
Fig. 3 Heatmap of the differentially expressed genes at FDR $<$ 0.05. The gene names were color coded according to the cell type in which they were found to differentially expressed (Red: B cells, Sage: CD4T, Green: CD8T, Turquoise: monocytes, Sky blue: PBMC, purple: whole blood). The last three genes in dark blue color corresponded to the differentially expressed genes identified by Neylan et al. ${ }^{32}$. The number printed in each heatmap cell corresponded to the estimated unadjusted $p$-value from DESeq2, and the color corresponded to the magnitude of the estimated log 2 fold change (blue: downregulated in PTSD, red: upregulated in PTSD)

\section{Discussion}

In order to enhance our understanding of gene regulation in PTSD, the current study was the first to characterize the gene expression profiles of four immune cell subsets (CD4T, CD8T, B cells, and monocytes) simultaneously using transcriptome-wide RNA-Seq. The objective of this study was to determine which immune cell subsets contributed most prominently to the transcriptomic changes in whole blood from our previous study $^{15}$. This study is the first to demonstrate an overlap as well as meaningful distinctions between different immune cell types implicated in differential gene expression in PTSD. Results revealed moderate correlations across the cell types for the 100 differentially expressed genes, with CD4T and CD8T being most similar to one another. Candidate gene analyses replicated earlier work identifying under-expression of PF4 in monocytes as linked to PTSD. Gene expression differences in PTSD were largest and most distinct in monocytes. Monocytes are the most common type of leukocyte, and play a role in adaptive immunity ${ }^{54}$. Circulating blood monocytes are also precursors of macrophages. Activated macrophages and monocytes have been proposed to play a major role in pathogenesis of several central nervous system conditions and neurological disorders as well as PTSD $^{55,56}$. Practically, these results suggest that future PTSD studies that wish to focus on gene expression in an isolated cell type might benefit from focusing on monocytes.

While our results replicated the upregulation in the candidate gene PF4 reported by Neylan et al. ${ }^{32}$, the findings did not replicate the predominant downregulation of gene expression in monocytes in PTSD. The difference could be attributed to the specific threshold used in Neylan et al. $^{32}$ for selecting candidate genes and the technical differences in assay systems. That is, we used RNA-Seq to establish the transcriptome profile, whereas Neylan et al. used microarrays. Furthermore, we considered a sequence of nominal $p$-value thresholds to ensure robustness of the results.

$F K B P 5$, a gene that plays a role in the regulation of the glucocorticoid receptor and immunological responses to 

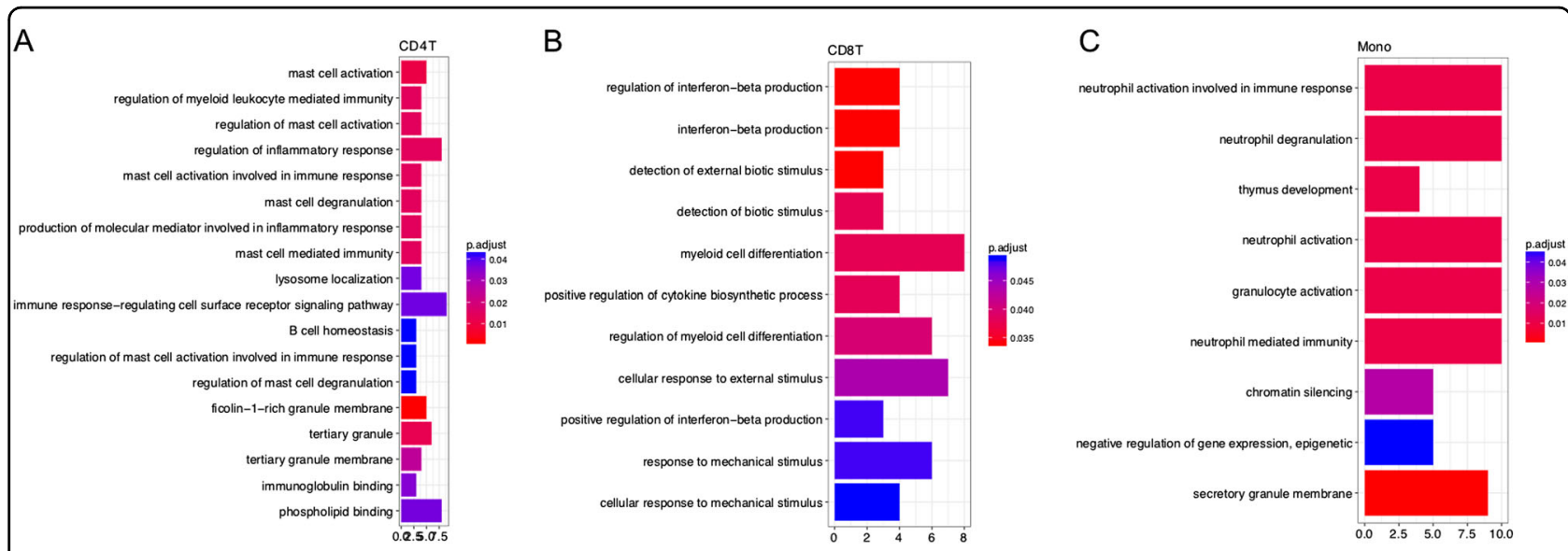

Fig. 4 Statistically significant gene ontologies at FDR $<0.05$ among the top 100 genes identified to be differentially expressed in PTSD for each cell type. $x$-axis denoted the number of differentially expressed genes overlapping with each gene ontology. No gene set was significant at $\mathrm{FDR}<0.05$ for $\mathrm{B}$ cells

stress, was previously found to be upregulated in whole blood $^{15}$. This study extended these results to show that the gene remained consistently upregulated in CD4T, CD8T, B cells, and monocytes, indicating that the effect of PTSD on FKBP5 may not be cell specific. This study also identified another gene (PI4KAP1) that was consistently upregulated in CD4T $(\mathrm{FDR}<0.05)$ and showed consistent trends across other cell types (nominal $p<0.05$ ). Both PI4KAP1 and PI4KAP2 are two non-processed pseudogene partial copies of $P I 4 K A$, a lipid kinase and proteincoding gene. PI4KAP2 was also consistently upregulated in all immune cell types, unsorted PBMC and whole blood $(p<0.05$ in B cells and CD4T), whereas PI4KA was marginally upregulated in all cell types except monocytes in this study. These results suggest that the upregulations of FKBP5 and PI4KAP1 appear to be important targets for future study.

Prior studies have found a significant association between PTSD and onset of neurodegenerative diseases $^{3,4}$. Several genes that were identified to be differentially expressed in the immune cell subsets have been implicated in neurodegenerative disorders. Despite the limited evidence regarding PI $4 K A P 1$, its parent gene, $P I 4 K A$, was implicated in late onset Alzheimer's disease via its role in the synthesis of phosphatidylinositol phosphate $^{57-59}$, which plays a diverse role in cell growth, differentiation and the nervous system ${ }^{60,61}$; whereas, the pseudogene PI4KAP2 was found to be dysregulated in Huntington's disease ${ }^{62}$. Both PI4KAP1 and PI4KAP2 are two non-processed pseudogene partial copy of PI4KA, and the presence of pseudogenes have been shown to complicate molecular genetic studies ${ }^{63,64}$. Pi4KAP2 and $P I 4 K A$ were also upregulated in all cell types (except PI4KA in monocytes), although it did not reach statistical significance. REST gene was significantly downregulated in monocytes comparing PTSD to control. REST gene is associated with neurodegeneration and has been identified as a master regulator of genes involved in neurogenesis and neuronal differentiation ${ }^{65,66}$. This gene was also found to be substantially reduced in individuals with mild cognitive impairment and almost absent in those with Alzheimer's disease ${ }^{67}$. Another gene SEPT4 identified to be significantly downregulated in monocytes, played a role in synaptic plasticity and neurodegeneration $^{68}$, and has been found to be downregulated in the amygdala and the substantia nigra of patients with Parkinson's disease ${ }^{69}$. This is particularly significant in our population where we recently demonstrated that our patients with PTSD have an increased risk of cognitive impairment $^{70}$, worse cognitive dysfunction across domains linked with neurodegenerative diseases ${ }^{71}$, and reduced functioning in neurologically associated mobility conditions $^{72}$.

The differentially expressed genes in PTSD identified within each cell type were enriched in gene sets related to immune response and inflammation. For example, gene sets in CD4T were involved in regulation of mast cells. Mast cells are the master regulators in immune response, and previous studies found that they may play an important role in innate and adaptive immune responses to inflammation and autoimmune diseases ${ }^{73-76}$. Mast cell activation in PTSD has been proposed to accelerate the pathogenesis of neurodegenerative diseases, including Alzheimer's disease ${ }^{77}$. Furthermore, regulation of interferon-beta production emerged as the top gene sets in CD8T cells. Interferon-beta is involved in a wide range of biological activities in human immune system, including activating cytotoxic effector cells ${ }^{78}$, acting as antimicrobial agent ${ }^{79}$, and promoting maturation of leukocytes ${ }^{80}$. Our finding is in line with emerging 
evidence that interferon-beta is also implicated in several autoimmune diseases, including multiple sclerosis and Alzheimer's disease $\mathrm{e}^{81-83}$, whereas interferon signaling, particularly interferon-gamma has been shown to be associated with PTSD $^{84,85}$. It is important to note that the pathway analyses were conducted on the top 100 genes ranked by the nominal $p$-values from differential expression analysis, where most of the genes did not reach statistical significance at $\mathrm{FDR}<0.05$. Thus, pending replication in independent samples, one should interpret the results from the pathway analyses with caution.

\section{Strengths and limitations}

The current study had several strengths, including the first to profile gene expression in isolated immune cell types in PTSD using the cutting-edge RNA-Seq. Nonetheless, our findings must be considered in the context of several limitations. First, these data represent information from a cross-sectional study with a relatively small sample size selected based on our previous polygenic gene expression scores from whole-blood transcriptome study $^{15}$, and thus we were not able to conclude whether immune cell subsets profiling is superior over whole blood. However, despite the small sample size, we were able to detect differentially expressed genes after adjusting for multiple comparisons for each cell type. The average percentage purity of the isolated immune cell subsets was $>93 \%$ (Supplementary Materials), suggesting that the identified genes were likely not artefact of mixed signals arising from contamination of other cell types during sorting. Nevertheless, further work is needed that seeks to replicate these analyses in a larger sample. Second, this study only profiled gene expression in four types of immune cells, namely CD4T, CD8T, B cells, and monocytes, and identified distinct differential gene expression patterns comparing WTC-PTSD to non-PTSD. Future studies should investigate other blood cell type subsets including natural killer cells, neutrophils, and eosinophils. Furthermore, it is unclear to what extent results generalize to other traumatized samples and to females. Finally, some of the cell subsets exist in very small fractions, which may not yield sufficient number of cells for the bulk RNA-Seq technology. Future studies should examine these cell subsets using the single cell sequencing technology.

\section{Conclusion}

The current study identified common and distinct gene signatures associated with PTSD in four subsets of leukocytes, indicating that the cell subpopulations may provide a valuable and to some degree independent source of information to refine the biomarker signature obtained from whole blood. In particular, monocytes showed the most differential gene expression in PTSD, suggesting that future work in isolated blood cells could focus on this subpopulation. Together with the results from pathway analysis, the gene expression profiles from cell subpopulations tentatively point to neurodegenerative mechanisms and to dysregulation in immune response. If independently replicated, these results add to a growing evidence suggesting that intervention strategies that target inflammatory responses may help to alleviate PTSD symptoms and related diseases.

\section{Data availability}

The RNA-Seq data of the 39 samples will be available at the Gene Expression Omnibus (accession number GSE114407) upon publication.

\section{Acknowledgements}

The study was funded by the Centers for Disease Control and Prevention (CDC)/The National Institute for Occupational Safety and Health (NIOSH) awards U01 OH010416-01 (PI: B.J.L.) and U01 OH011478-01 (PI: P.F.K.). We gratefully acknowledge the support of the first responders and rescue/ recovery workers for participating in this study. We also thank the staff of the Stony Brook World Trade Center Health Program and the World Trade Center Health Program Data Monitoring Center for ongoing support. RNA extraction and RNA-seq was performed at the Roswell Park Roswell Park Comprehensive Cancer Center Genomics Shared Resource facility supported by National Cancer Institute (NCl) grant P30CA016056. The findings and conclusions in this article are those of the authors and do not represent the official position of $\mathrm{NIOSH}$, the CDC, or the US Public Health Service.

\section{Author details \\ ${ }^{1}$ Department of Applied Mathematics and Statistics, Stony Brook University, Stony Brook, NY, USA. ²Department of Medicine, Stony Brook University, Stony Brook, NY, USA. ${ }^{3}$ Department of Family and Preventive Medicine, Stony Book University, Stony Brook, NY, USA. ${ }^{4}$ Department of Psychiatry, Stony Book University, Stony Brook, NY, USA. ${ }^{5}$ Center for Personalized Medicine, Roswell Park Comprehensive Cancer Center, Buffalo, NY, USA. ${ }^{6}$ Department of Biostatistics and Bioinformatics, Roswell Park Comprehensive Cancer Center, Buffalo, NY, USA}

Conflict of interest

The authors declare that they have no conflict of interest.

\section{Publisher's note}

Springer Nature remains neutral with regard to jurisdictional claims in published maps and institutional affiliations.

Supplementary Information accompanies this paper at (https://doi.org/ 10.1038/s41398-018-0355-8).

Received: 15 May 2018 Revised: 26 November 2018 Accepted: 10 December 2018

Published online: 15 January 2019

\section{References}

1. Kessler, R. C. et al. Lifetime prevalence and age-of-onset distributions of DSMIV disorders in the National Comorbidity Survey Replication. Arch. Gen. Psychiatry 62, 593-602 (2005).

2. Association, A. P. Diagnostic and statistical manual of mental disorders: DSM-5. 5th edn, (American Psychiatric Association, Washington, DC, 2013).

3. Greenberg, M. S., Tanev, K., Marin, M. F. \& Pitman, R. K. Stress, PTSD, and dementia. Alzheimers Dement. 10(3 Suppl), S155-S165 (2014).

4. Yaffe, $\mathrm{K}$. et al. Posttraumatic stress disorder and risk of dementia among US veterans. Arch. Gen. Psychiatry 67, 608-613 (2010).

5. Kessler, R. C. Posttraumatic stress disorder: the burden to the individual and to society. J. Clin. Psychiatry 61(Suppl 5), 4-12 (2000). discussion13-14. 
6. Cornelis, M. C., Nugent, N. R., Amstadter, A. B. \& Koenen, K. C. Genetics of posttraumatic stress disorder: review and recommendations for genome-wide association studies. Curr. Psychiatry Rep. 12, 313-326 (2010).

7. Stein, M. B., Jang, K. L., Taylor, S., Vernon, P. A. \& Livesley, W. J. Genetic and environmental influences on trauma exposure and posttraumatic stress disorder symptoms: a twin study. Am. J. Psychiatry 159, 1675-1681 (2002).

8. True, W. R. et al. A twin study of genetic and environmental contributions to liability for posttraumatic stress symptoms. Arch. Gen. Psychiatry 50, 257-264 (1993).

9. Koenen, K. C. Genetics of posttraumatic stress disorder: review and recommendations for future studies. J. Trauma. Stress 20, 737-750 (2007).

10. Passos, I. C. et al. Inflammatory markers in post-traumatic stress disorder: a systematic review, meta-analysis, and meta-regression. Lancet Psychiatry $\mathbf{2}$ 1002-1012 (2015).

11. Michopoulos, V., Powers, A., Gillespie, C. F., Ressler, K. J. \& Jovanovic, T. Inflammation in fear-and anxiety-based disorders: PTSD, GAD, and beyond. Neuropsychopharmacology 42, 254-270 (2016).

12. Rosen, R. L. et al. Elevated C-reactive protein and posttraumatic stress pathology among survivors of the 9/11 World Trade Center attacks. J. Psychiatr. Res. 89, 14-21 (2017).

13. Logue, M. W. et al. An analysis of gene expression in PTSD implicates genes involved in the glucocorticoid receptor pathway and neural responses to stress. Psychoneuroendocrinology 57, 1-13 (2015).

14. Kukurba, K. R. \& Montgomery, S. B. RNA sequencing and analysis. Cold Spring Harb. Protoc. 2015, 951-969 (2015)

15. Kuan, P. F. et al. Gene expression associated with PTSD in World Trade Center responders: an RNA sequencing study. Transl. Psychiatry 7, 1297 (2017).

16. Bam, M. et al. Dysregulated immune system networks in war veterans with PTSD is an outcome of altered miRNA expression and DNA methylation. Sci. Rep. 6, 31209 (2016)

17. Breen, M. S. et al. Gene networks specific for innate immunity define posttraumatic stress disorder. Mol. Psychiatry 20, 1538-1545 (2015).

18. Guardado, P. et al. Altered gene expression of the innate immune, neuroendocrine, and nuclear factor-kappa B (NF-kB) systems is associated with posttraumatic stress disorder in military personnel. J. Anxiety Disord. 38, 9-20 (2016).

19. Sarapas, C. et al. Genetic markers for PTSD risk and resilience among survivors of the World Trade Center attacks. Dis. Markers 30, 101-110 (2011).

20. Segman, R. et al. Peripheral blood mononuclear cell gene expression profiles identify emergent post-traumatic stress disorder among trauma survivors. Mol Psychiatry 10, 500-513 (2005)

21. Yehuda, R. et al. Gene expression patterns associated with posttraumatic stress disorder following exposure to the World Trade Center attacks. Biol. Psychiatry 66, 708-711 (2009).

22. Mehta, D. et al. Childhood maltreatment is associated with distinct genomic and epigenetic profiles in posttraumatic stress disorder. Proc. Natl Acad. Sci. USA 110, 8302-8307 (2013).

23. Sullivan, P. F., Fan, C. \& Perou, C. M. Evaluating the comparability of gene expression in blood and brain. Am. J. Med. Genet. B Neuropsychiatr. Genet. 141B, 261-268 (2006)

24. Glatt, S. J. et al. Comparative gene expression analysis of blood and brain provides concurrent validation of SELENBP1 up-regulation in schizophrenia. Proc. Natl Acad. Sci. USA 102, 15533-15538 (2005).

25. Becker, A. M. et al. SLE peripheral blood B cell, $T$ cell and myeloid cell transcriptomes display unique profiles and each subset contributes to the interferon signature. PLOS ONE 8, e67003 (2013).

26. McKinney, E. F., Lee, J. C., Jayne, D. R., Lyons, P. A. \& Smith, K. G. T-cell exhaustion, co-stimulation and clinical outcome in autoimmunity and infection. Nature 523, 612-616 (2015).

27. McKinney, E. F. et al. A CD8+ T cell transcription signature predicts prognosis in autoimmune disease. Nat. Med. 16, 586-591 (2010). 581p following 591.

28. O'Donovan, A. et al. Elevated risk for autoimmune disorders in iraq and afghanistan veterans with posttraumatic stress disorder. Biol. Psychiatry 77, 365-374 (2015).

29. Wang, Z. \& Young, M. R. PTSD, a disorder with an immunological component. Front. Immunol. 7, 219 (2016).

30. Webber, M. P. et al. Post-September 11, 2001, incidence of systemic autoimmune diseases in World Trade Center-exposed firefighters and emergency medical service workers. Mayo Clin. Proc. 91, 23-32 (2016).
31. Webber, M. P. et al. Nested case-control study of selected systemic autoimmune diseases in World Trade Center rescue/recovery workers. Arthritis Rheumatol. 67, 1369-1376 (2015).

32. Neylan, T. C. et al. Suppressed monocyte gene expression profile in men versus women with PTSD. Brain Behav. Immun. 25, 524-531 (2011).

33. Kotov, R. et al. Posttraumatic stress disorder and the risk of respiratory problems in World Trade Center responders: longitudinal test of a pathway. Psychosom. Med. 77, 438-448 (2015).

34. Bromet, E. et al. DSM-IV post-traumatic stress disorder among World Trade Center responders 11-13 years after the disaster of 11 September 2001 (9/11) Psychol. Med. 46, 771-783 (2016)

35. Dasaro, C. R. et al. Cohort Profile: World Trade Center Health Program General Responder Cohort. Int. J. Epidemiol. 46, dyv099 (2015).

36. First, M. B., Spitzer, R. L., Gibbon, M. \& Williams, J. B. Structured Clinical Interview for DSM-IV Axis I Disorders: Patient Edition (February 1996 Final), SCID-I/P. (Biometrics Research Department, New York State Psychiatric Institute, 1998).

37. Mallone, R. et al. Isolation and preservation of peripheral blood mononuclear cells for analysis of islet antigen-reactive T cell responses: position statement of the T-Cell Workshop Committee of the Immunology of Diabetes Society. Clin. Exp. Immunol. 163, 33-49 (2011).

38. Kim, D. et al. TopHat2: accurate alignment of transcriptomes in the presence of insertions, deletions and gene fusions. Genome Biol. 14, 1 (2013).

39. Langmead, B. \& Salzberg, S. L. Fast gapped-read alignment with Bowtie 2. Nat. Methods 9, 357-359 (2012).

40. Pruitt, K. D., Tatusova, T. \& Maglott, D. R. NCBI reference sequences (RefSeq): a curated non-redundant sequence database of genomes, transcripts and proteins. Nucleic Acids Res. 35(suppl 1), D61-D65 (2007).

41. Rosenbloom, K. R. et al. The UCSC genome browser database: 2015 update. Nucleic Acids Res. 43(D1), D670-D681 (2015).

42. Andrews, S. FastQC: A quality control tool for high throughput sequence data. Reference Source 2010. https://www.bioinformatics.babraham.ac.uk/projects/ fastqc

43. Martin, M. Cutadapt removes adapter sequences from high-throughput sequencing reads. EMBnet J. 17, 10-12 (2011).

44. Anders, S., Pyl, P. T. \& Huber, W. HTSeq-a Python framework to work with high-throughput sequencing data. Bioinformatics 31, btu638 (2014).

45. Leek, J. T. Svaseq: removing batch effects and other unwanted noise from sequencing data. Nucleic Acids Res. 42(21), e161 (2014).

46. Newman, A. M. et al. Robust enumeration of cell subsets from tissue expression profiles. Nat. Methods 12, 453-457 (2015)

47. Aran, D., Hu, Z. \& Butte, A. J. xCell: digitally portraying the tissue cellular heterogeneity landscape. Genome Biol. 18, 220 (2017).

48. Love, M. I., Huber, W. \& Anders, S. Moderated estimation of fold change and dispersion for RNA-seq data with DESeq2. Genome Biol. 15, 1 (2014).

49. Robinson, M. D., McCarthy, D. J. \& Smyth, G. K. edgeR: a Bioconductor package for differential expression analysis of digital gene expression data. Bioinformatics 26, 139-140 (2010).

50. Benjamini, Y. \& Hochberg, Y. Controlling the false discovery rate: a practical and powerful approach to multiple testing. J. R. Stat. Soc. B 57, 289-300 (1995).

51. Yu, G., Wang, L.-G., Han, Y. \& He, Q.-Y. clusterProfiler: an R package for comparing biological themes among gene clusters. OMICS 16, 284-287 (2012).

52. Zou, H. \& Hastie, T. Regularization and variable selection via the elastic net. J. $R$ Stat. Soc.: Ser. B 67, 301-320 (2005).

53. Langfelder, P. \& Horvath, S. WGCNA: an R package for weighted correlation network analysis. BMC Bioinform. 9, 559 (2008).

54. Leon, B. \& Ardavin, C. Monocyte-derived dendritic cells in innate and adaptive immunity. Immunol. Cell Biol. 86, 320-324 (2008).

55. Bam, M. et al. Decreased AGO2 and DCR1 in PBMCs from War Veterans with PTSD leads to diminished miRNA resulting in elevated inflammation. Transl. Psychiatry 7, e1222 (2017).

56. Williams, K. C. \& Hickey, W. F. Central nervous system damage, monocytes and macrophages, and neurological disorders in AIDS. Annu. Rev. Neurosci. 25, 537-562 (2002)

57. Bothmer, J., Markerink, M. \& Jolles, J. Phosphoinositide kinase activities in synaptosomes prepared from brains of patients with Alzheimer's disease and controls. Neurosci. Lett. 176, 169-172 (1994).

58. Stokes, C. E. \& Hawthorne, J. N. Reduced phosphoinositide concentrations in anterior temporal cortex of Alzheimer-diseased brains. J. Neurochem. 48, 1018-1021 (1987). 
59. Rodriguez-Seijas, C., Stohl, M., Hasin, D. S. \& Eaton, N. R. Transdiagnostic factors and mediation of the relationship between perceived racial discrimination and mental disorders. JAMA Psychiatry 72, 706-713 (2015).

60. Clayton, E. L., Minogue, S. \& Waugh, M. G. Phosphatidylinositol 4-kinases and PI4P metabolism in the nervous system: roles in psychiatric and neurological diseases. Mol. Neurobiol. 47, 361-372 (2013).

61. Kim, S. K., Kim, H., Yang, Y. R., Suh, P. G. \& Chang, J. S. Phosphatidylinositol phosphates directly bind to neurofilament light chain (NF-L) for the regulation of NF-L self assembly. Exp. Mol. Med. 43, 153-160 (2011).

62. Costa, V., Esposito, R., Aprile, M. \& Ciccodicola, A. Non-coding RNA and pseudogenes in neurodegenerative diseases: "The (un)Usual Suspects". Front. Genet. 3, 231 (2012).

63. Pagnamenta, A. T. et al. Germline recessive mutations in PI4KA are associated with perisylvian polymicrogyria, cerebellar hypoplasia and arthrogryposis. Hum. Mol. Genet. 24, 3732-3741 (2015).

64. Ziyad, S. et al. A forward genetic screen targeting the endothelium reveals a regulatory role for the lipid kinase Pi4ka in Myelo- and erythropoiesis. Cell Rep. 22, 1211-1224 (2018).

65. Hwang, J. Y. \& Zukin, R. S. REST, a master transcriptional regulator in neurodegenerative disease. Curr. Opin. Neurobiol. 48, 193-200 (2018).

66. Noh, K. M. et al. Repressor element-1 silencing transcription factor (REST)dependent epigenetic remodeling is critical to ischemia-induced neuronal death. Proc. Natl Acad. Sci. USA 109, E962-E971 (2012).

67. Whalley, K. Neurodegeneration: ageing neurons need REST. Nat. Rev. Neurosci. 15, 279 (2014).

68. Marttinen, M., Kurkinen, K. M., Soininen, H., Haapasalo, A. \& Hiltunen, M. Synaptic dysfunction and septin protein family members in neurodegenerative diseases. Mol. Neurodegener. 10, 16 (2015).

69. Shehadeh, L., Mitsi, G., Adi, N., Bishopric, N. \& Papapetropoulos, S. Expression of Lewy body protein septin 4 in postmortem brain of Parkinson's disease and control subjects. Mov. Disord. 24, 204-210 (2009).

70. Clouston, S. A. et al. Cognitive impairment among World Trade Center responders: Long-term implications of re-experiencing the $9 / 11$ terrorist attacks. Alzheimers Dement 4, 67-75 (2016).

71. Clouston, S. et al. Traumatic exposures, posttraumatic stress disorder, and cognitive functioning in World Trade Center responders. Alzheimers Dement $\mathbf{3}$, 593-602 (2017).
72. Clouston, S. A. P., Guralnik, J. M., Kotov, R., Bromet, E. J. \& Luft, B. J. Functional limitations among responders to the World Trade Center attacks 14 years after the disaster: implications of chronic posttraumatic stress disorder. J. Trauma. Stress 30, 443-452 (2017).

73. Abraham, S. N. \& St John, A. L. Mast cell-orchestrated immunity to pathogens. Nat. Rev. Immunol. 10, 440-452 (2010).

74. Gilfillan, A. M. \& Beaven, M. A. Regulation of mast cell responses in health and disease. Crit. Rev. Immunol. 31, 475-529 (2011).

75. Metz, M., Siebenhaar, F. \& Maurer, M. Mast cell functions in the innate skin immune system. Immunobiology 213, 251-260 (2008).

76. Galli, S. J., Maurer, M. \& Lantz, C. S. Mast cells as sentinels of innate immunity. Curr. Opin. Immunol. 11, 53-59 (1999).

77. Kempuraj, D. et al. Mast cell activation in brain injury, stress, and post-traumatic stress disorder and Alzheimer's disease pathogenesis. Front. Neurosci. 11, 703 (2017).

78. Kito, T., Kuroda, E., Yokota, A. \& Yamashita, U. Enhancement of macrophage cytotoxicity against murine gliomas by interferon beta: increase in nitric oxide production in response to glioma-derived soluble factors. J. Neurosurg. 97, 619-626 (2002)

79. Ito, T. et al. Differential regulation of human blood dendritic cell subsets by IFNs. J. Immunol. 166, 2961-2969 (2001).

80. Nguyen, K. B. et al. Coordinated and distinct roles for IFN-alpha beta, IL-12, and IL-15 regulation of NK cell responses to viral infection. J. Immunol. 169, 4279-4287 (2002).

81. Pena Rossi, C. et al. Interferon beta-1a for the maintenance of remission in patients with Crohn's disease: results of a phase II dose-finding study. BMC Gastroenterol. 9, 22 (2009).

82. Sin, W. X., Li, P., Yeong, J. P. \& Chin, K. C. Activation and regulation of interferonbeta in immune responses. Immunol. Res. 53, 25-40 (2012).

83. Grimaldi, L. M. E. et al. A pilot study on the use of interferon beta-1a in early Alzheimer's disease subjects. J. Neuroinflammation 11, 30 (2014).

84. Bruenig, D. et al. Correlation between interferon $\gamma$ and interleukin 6 with PTSD and resilience. Psychiatry Res. 260, 193-198 (2018).

85. Griffin, G. D., Charron, D. \& Al-Daccak, R. Post-traumatic stress disorder: revisiting adrenergics, glucocorticoids, immune system effects and homeostasis. Clin. Transl. Immunol. 3, e27 (2014). 